\title{
Critical Phenomena Associated with Boson Stars
}

\author{
Scott H. Hawley* and Matthew W. Choptuik ${ }^{\dagger}$ \\ * Max Planck Institut für Gravitationsphysik, Albert Einstein Institut, 14476 Golm, Germany \\ ${ }^{\dagger}$ CIAR Cosmology and Gravity Program, Department of Physics and Astronomy, University of \\ British Columbia, Vancouver, British Columbia, Canada V6T 1Z1
}

Here we present a synopsis of related work [1,2] describing a study of black hole threshold phenomena for a self-gravitating massive complex scalar field in spherical symmetry.

Studies of models of gravitational collapse have revealed structure which can arise near the threshold of black hole formation. The solutions in this regime are known as "critical solutions," and their properties as "critical phenomena". These solutions can arise generically, even in simple models such a massless scalar field in spherical symmetry [3].

Critical solutions can be constructed dynamically via numerical simulations, in which one considers continuous one-parameter families of initial data with the following "interpolating" property: for sufficiently large values of the family parameter, $p$, the evolved data describes a spacetime containing a black hole, whereas for sufficiently small values of $p$, the matter-energy in the spacetime disperses to large radii at late times, and no black hole forms. Within this range of parameters, there will exist a critical parameter value, $p=p^{\star}$, which demarks the onset, or threshold, of black hole formation.

Over the past decade, numerical and closed-form studies of collapse in various matter models have enlarged the picture of critical phenomena $[4,5,6,7]$, so that we now have a more complete understanding of the relevant dynamics. (Interested readers should consult the reviews by Gundlach [8,9] for a more comprehensive discussion of critical phenomena.) Black-hole-threshold solutions are attractors in the sense that they are almost completely independent of the specifics of the particular family used as a generator. Up to the current time, the only initial data dependence which has been observed in critical collapse occurs in models for which there is more than one distinct black-hole-threshold solution. Critical solutions are by construction unstable, having precisely one unstable mode $[10,11]$. Thus letting $p \rightarrow p^{\star}$ amounts to minimizing or "tuning away" the initial amplitude of the unstable mode present in the system. These solutions also possess additional symmetry which, to date, has either been a time-translation symmetry, in which the critical solution is static or periodic, or a scale-translation symmetry (hometheticity), in which the critical solution is either continuously or discretely self-similar (CSS or DSS).

These symmetries are indicative of the two principal types of critical behavior that have been seen in black hole threshold studies (with some models exhibiting both types of behavior depending on the initial data). For Type I solutions, there is a finite minimum black hole mass which can be formed, and there exists a scaling law for the lifetime $\tau$ of the near-critical solutions such that $\tau \sim-\gamma \ln \left|p-p^{\star}\right|$ where $\gamma$ is a model-specific exponent. In Type II critical behavior, a black hole of arbitrarily small mass can be formed, and the critical solutions are generically self-similar. 


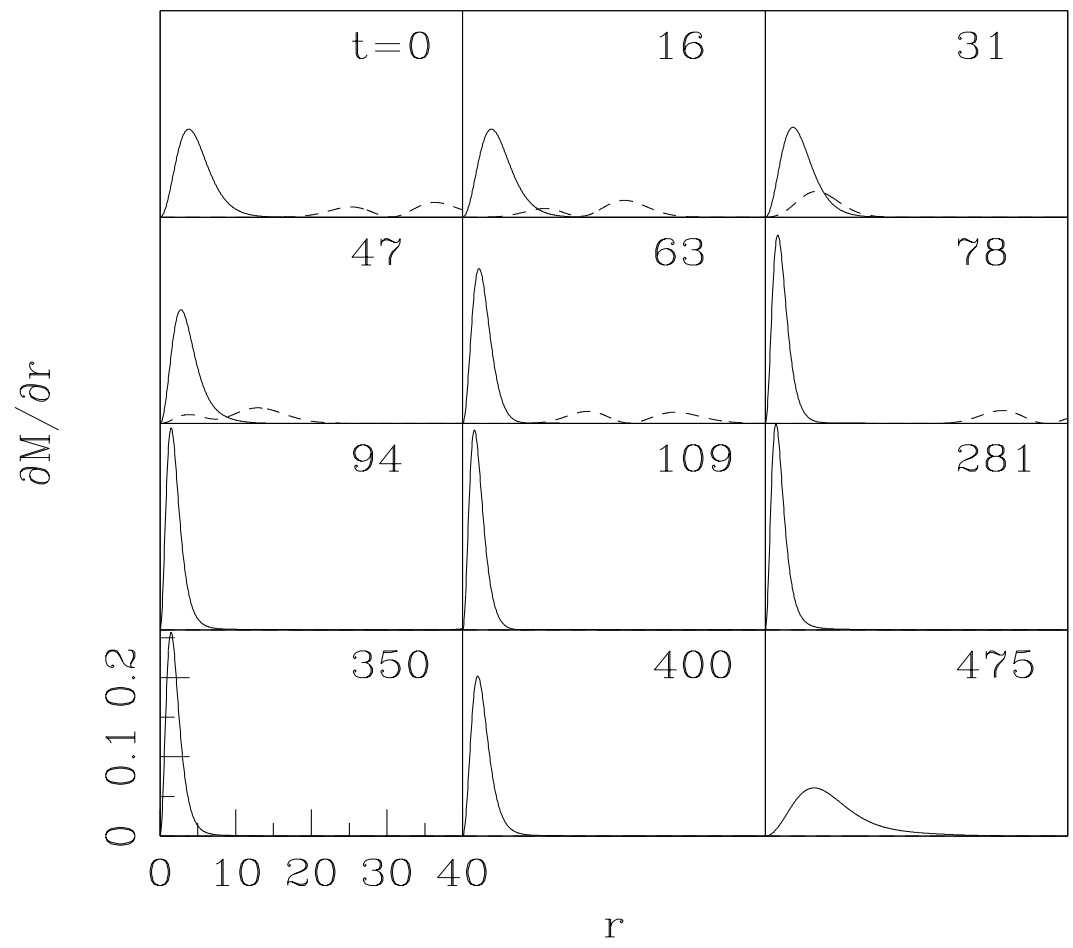

FIGURE 1. Evolution of a perturbed stable boson star with $\phi_{0}(0)=0.04 \times \sqrt{4 \pi}$ and mass $M_{C}=$ $0.59 M_{P l}^{2} / m$. This shows contributions to the radial derivative of the total mass $\partial M(t, r) / \partial r$ due to the massive field $\phi(t, r)$ (solid line) and massless field $\phi_{3}(t, r)$ (dashed line). We start with a stable boson star centered at the origin, and a gaussian pulse of massless field. (We see two peaks for the massless field because it is only the gradients of $\phi_{3}$, not $\phi_{3}$ itself, which contribute to $\partial M / \partial r$.) In the evolution shown above, the pulse of massless field enters the region containing the bulk of the boson star $(t \simeq 15)$, implodes through the origin $(t \simeq 30)$ and leaves the region of the boson star $(t \simeq 50)$. Shortly after the massless pulse passes through the origin, the boson star collapses into a more compact configuration, about which it oscillates for a long time before either forming a black hole or dispersing. (The case of dispersal is shown here.)

Our current interest is a critical-phenomena-inspired study of the dynamics associated with "boson stars" $[12,13,14,15,16]$. A boson star is given by a complex massive scalar field $\phi(t, r)=\phi_{0}(r) \exp (i \omega t)$, minimally coupled to gravity as given by general relativity. In this study, we dynamically construct critical solutions of the Einstein equations coupled to a massive, complex scalar field $\phi(t, r)$, by simulating the implosion of a spherical shell of massless real scalar field $\phi_{3}(t, r)$ around an "enclosed" boson star. The massless pulse then passes through the origin, explodes and continues to $r \rightarrow \infty$, while the massive complex (boson star) field is compressed into a state which ultimately either forms a black hole or disperses. For the massless field $\phi_{3}(0, r)$, we choose a gaussian of fixed width $\Delta$ and initial distance $r_{0}$ from the origin, and vary the amplitude $A$ until the critical solution is obtained (to within machine precision).

Figure 1 shows a series of snapshots from a typical simulation in which the parameter $p(p \equiv A)$ is slightly below the critical value $p^{\star}$, for an initial stable boson star with 


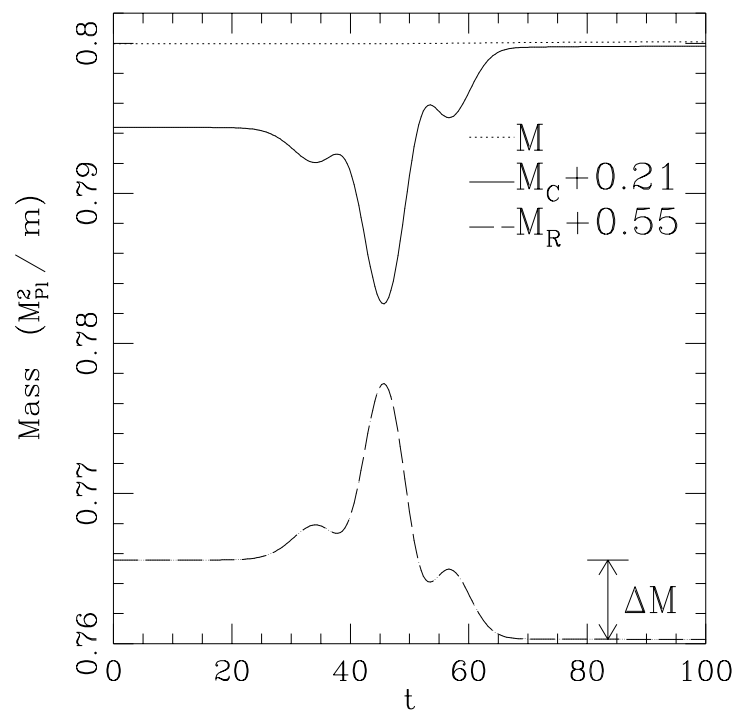

FIGURE 2. Exchange of energy between the real and complex scalar fields. The solid line shows the mass of the complex field, shifted upward by $0.21 M_{P l}^{2} / m$ for graphing purposes. The long-dashed line shows the mass of the real field, shifted upward by $0.55 M_{P l}^{2} / m$. The amount (and percentage) of mass transfer goes to zero as we consider boson star initial data approaching the maximum mass (the transition to instability). The dotted line near the top of the graph shows the total mass $M=M_{C}+M_{R}$, which is conserved to within a few hundredths of a percent. In all cases, we only see a net transfer of mass from the real field to to complex field, and not vice versa.

a mass of $M=0.59 M_{P l}^{2} / m$ (where $M_{P l}$ is the Planck mass). In this figure, we have plotted the individual contributions of the complex and real fields to the total mass of the spacetime. That is, we have defined masses $M_{C}$ and $M_{R}$ of the complex and real fields, respectively. (Only in vacuum regions and for times at which the supports of the two fields do not overlap can $M_{C}$ and $M_{R}$ be interpreted as physically meaningful masses.) During this gravitational "collision," mass is is transferred from the real to the complex field, as shown in Figure 2.

The resulting critical solutions persist for some finite amount of time which depends on the fine-tuning $p-p^{\star}$ of the initial data. As we have shown in [1], the lifetimes $\tau$ of the near-critical solutions follow the scaling law for Type I solutions, $\tau=-\gamma \ln \left|p-p^{\star}\right|$. Here $\gamma$ is related to the imaginary part of the growth factor $\sigma$ of the unstable mode $(\sim \exp [i \sigma t])$ by $\mathfrak{I}(\sigma)=1 / \gamma$. In keeping with the Type I nature of these solutions, we find a finite minimum mass for the black hole formed for as we let $p \rightarrow p^{\star}$ (for $p>p^{\star}$ ).

The critical solutions have properties which correspond closely with those of unstable boson stars, as shown in Figure 3. To further extend the comparison between these critical solutions and boson stars, we perform a linear perturbation analysis about boson star equilibria, building on the work of Gleiser and Watkins [17]. Using the method described in [1], we find the distribution for the squared frequency $\sigma^{2}$ of boson star quasinormal modes with respect to $\phi_{0}(0)$, and we find the radial shapes of the modes. In Figure 4 we provide a comparison between unstable modes found from our simulations and corresponding results obtained via perturbation theory about a boson star which 

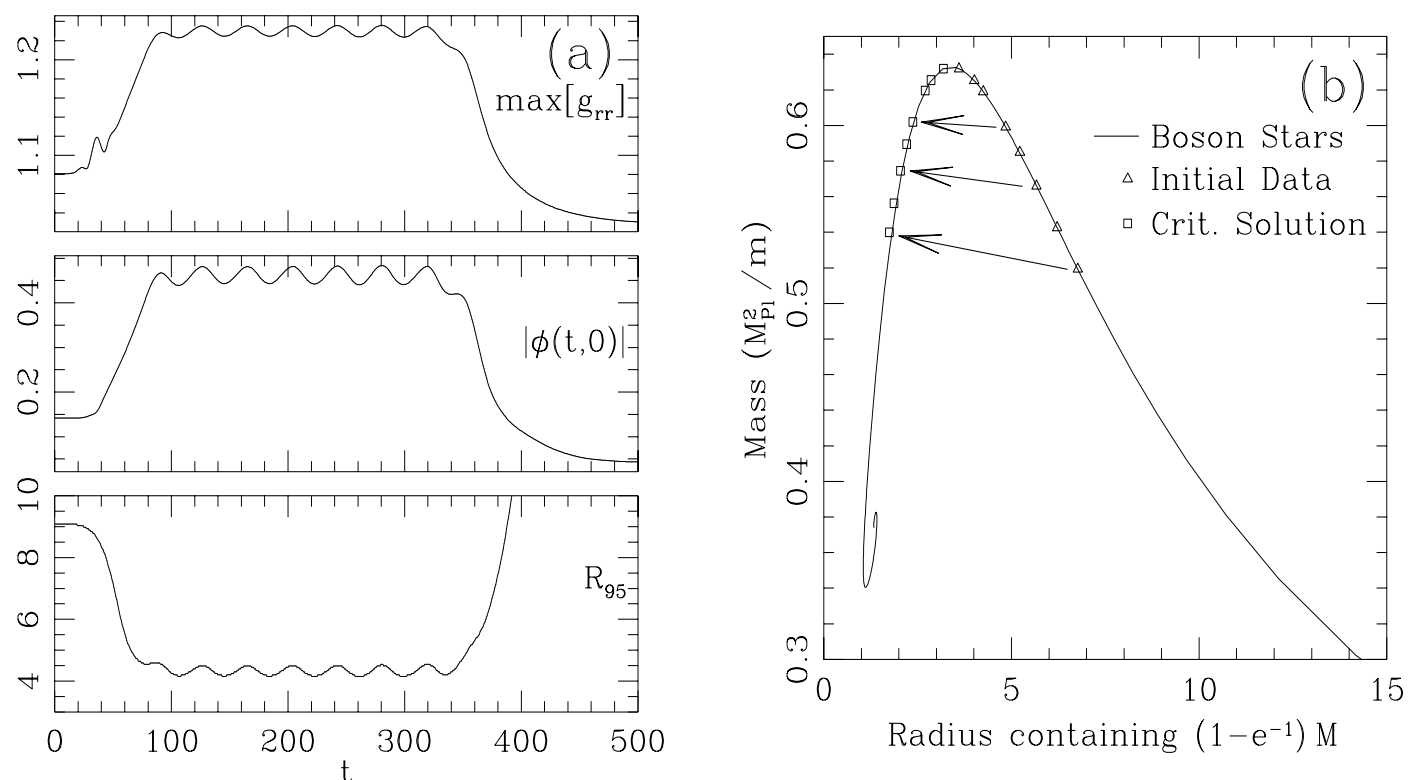

FIGURE 3. (a) Quantities describing a near-critical solution. Here we show timelike slices through the data shown in Figure 1, an evolution which ends in dispersal. Top: Maximum value of the metric function $g_{r r}(t, r)$. Middle: Central value $|\phi(t, 0)|$ of the massive field. Bottom: Radius $R_{95}$ containing $95 \%$ of the mass-energy in the complex field. (b) Mass $v s$. radius for equilibrium configurations of boson stars (solid line), initial data for the complex field (triangles), and critical solutions (squares). Arrows are given to help match initial data with the resulting critical solutions. Points on the solid line to the left of the maximum mass $M_{\max } \simeq 0.633 M_{P l}^{2} / m$ correspond to unstable boson stars, whereas those to the right of the maximum correspond to stable stars. The squares show the time average of each critical solution, which exists during the oscillatory regime shown in (a). We show the radius containing $\left(1-e^{-1}\right) M \simeq 0.63 M$ instead of $R_{95}$ in order to exclude the halo which forms in the critical solution (see Figure 5).

is a "best fit" to the simulation data. We find close agreement between the shapes and frequencies of the unstable modes obtained by these two different methods. A comparison of the next higher (oscillatory) mode also yields favorable results.

Thus the critical solutions we obtain appear to correspond to boson stars exhibiting superpositions of stable and unstable modes. For boson stars with masses somewhat less than the maximum boson star mass $M_{\max } \simeq 0.633 M_{P l}^{2} / m$ (e.g. those boson stars with masses $0.9 M_{\max }$ or less), however, we find less than complete agreement between the critical solutions and unstable boson stars of comparable mass. This is evidenced by the presence of an additional spherical shell or "halo" of matter in the critical solution, located in what would be the tail of the corresponding boson star. Interior to this halo, we find that the critical solution compares favorably with the boson star profile.

It is our contention that this halo is not part of the true critical solution, but is instead an artifact of the collision with the massless field. As one might expect, the properties of the halo are not universal, i.e. they are quite dependent on the type of initial data used. In contrast, the critical solution interior to the halo is largely independent of the form of the initial data. To demonstrate this, we use two families of initial data, given by a gaussian a "kink" $\left(\phi_{3}(0, r)=A / 2\left[1+\tanh \left[\left(r-r_{0}\right) / \Delta\right]\right)\right.$. A series of snapshots from one 

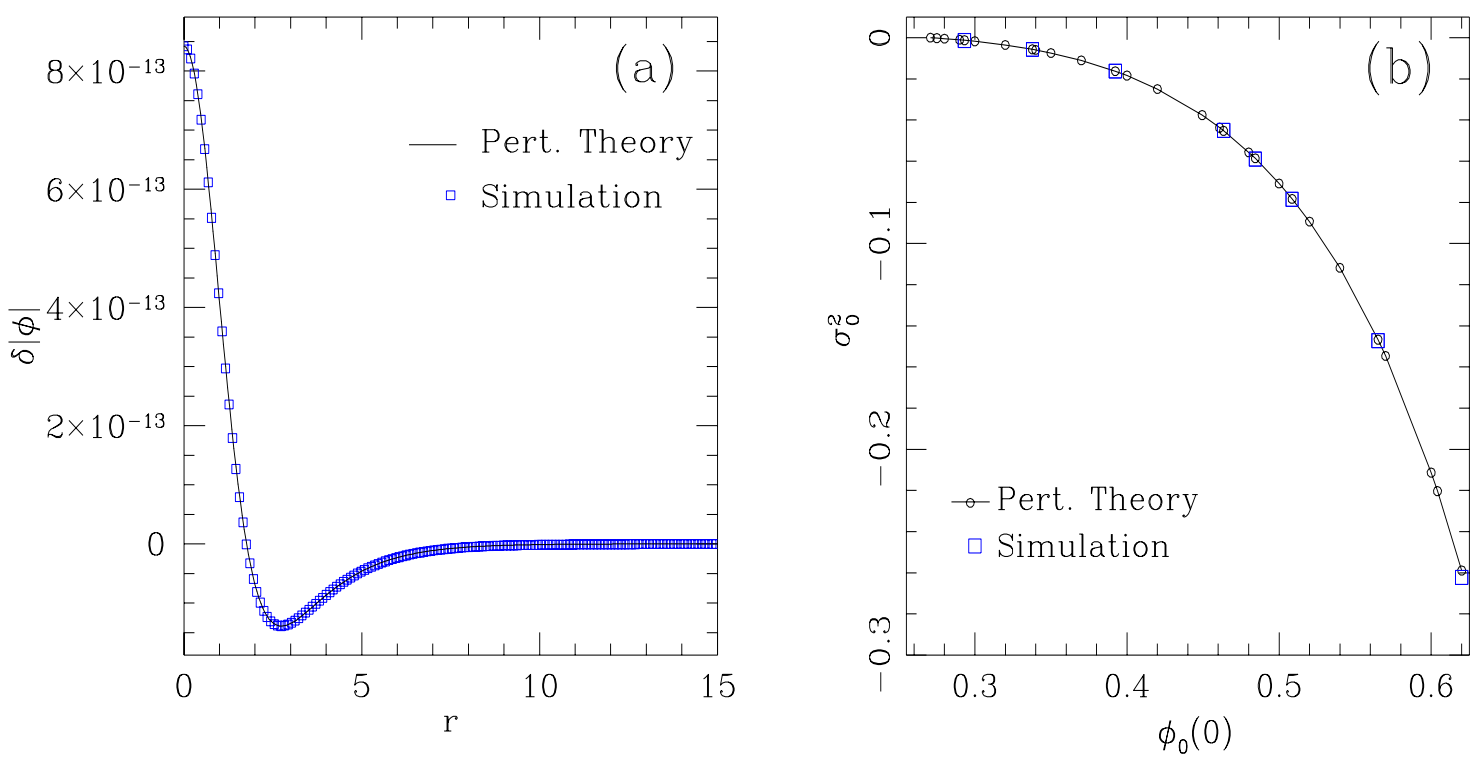

FIGURE 4. (a) Fundamental mode of unstable boson star. The solid line shows $\delta|\phi|$ obtained from the perturbation theory calculations. The squares show the difference between $|\phi|$ for two simulations for which the critical parameter $p$ differs by $10^{-14}$. Differences between the simulation data and perturbation theory results are below $1.1 \times 10^{-15}$. (b) Comparison of squared growth factors (squared Lyapunov exponents) $\sigma_{0}^{2}$ for unstable modes. The circles show a subset of the perturbation theory results we obtained for unstable boson stars. The squares show the measurements of unstable growth factors in our simulations. (The solid line simply connects the circles.)

such pair of evolutions is shown in Figure 5. We suspect that the halo is radiated over time (via scalar radiation, or "gravitational cooling" [18]) for all critical solutions. We find, however, that the time scale for radiation of the halo is comparable to the time scale for dispersal or black hole formation for each (nearly) critical solution we consider. With higher numerical precision, one might be able to more finely tune out the unstable mode, allowing more time to observe the behavior of the halo before dispersal or black hole formation occur.

For the future, we consider it worthwhile to investigate similar scenarios for neutron stars. While there have bee studies regarding the explosion of neutron stars near the minimum mass (e.g.,[19]), we would like to see whether neutron stars of non-minimal mass can be driven to explode via dispersal from a critical solution. 


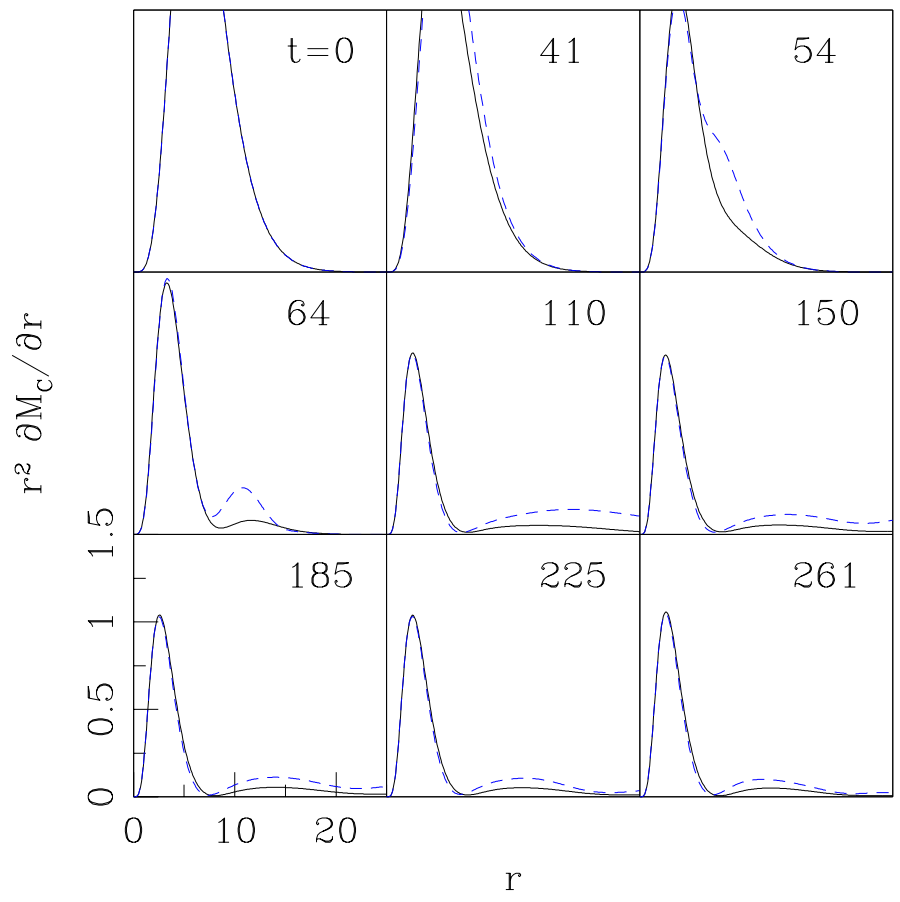

FIGURE 5. Evolution of $r^{2} \partial M_{C} / \partial r$ for for two different sets of initial data. Both sets contain the same initial boson star, but the massless field $\phi_{3}(0, r)$ (not shown) for one set is given by a gaussian whereas for the other set $\phi_{3}(0, r)$ is given by a "kink" . The amplitude of each pulse is varied (independently for each family of initial data) to obtain the critical solution. We have multiplied $\partial M_{C} / \partial r$ by $r^{2}$ to highlight the dynamics of the halo; thus the main body of the solution appears to decrease in size as it moves to lower values of $r$. The kink data produces a larger and much more dynamical halo, but interior to the halo, the two critical solutions match closely - and also match the profile of an unstable boson star. Thus, the portion of the solution which is "universal" corresponds to an unstable boson star. One can see the additional halo of matter in the region roughly $8 \leq r \leq 23$, exterior to the bulk of the critical solution.

\section{REFERENCES}

1. S.H. Hawley and M.W. Choptuik, Phys. Rev. D 62, 104024 (2000).

2. S.H. Hawley, Ph.D. Dissertation, University of Texas at Austin (2000).

3. M.W. Choptuik, Phys. Rev. Lett. 70, 9 (1993).

4. D.W. Neilsen and M.W. Choptuik, Class.Quant.Grav. 17, 761-782 (2000).

5. M.W. Choptuik, T. Chmaj and P. Bizon, Phys. Rev. Lett. 77, 424 (1996).

6. P.R. Brady, C.M. Chambers and S.M.C.V. Conçalves, Phys. Rev. D 56, 6057 (1997).

7. A.M. Abrahams and C.R. Evans, Phys. Rev. Lett. 70, 2980 (1993).

8. C. Gundlach, Adv. Theor. Math.Phys. 2, 1-49 (199 8).

9. C. Gundlach, Living Reviews 1999-4 (1999).

10. C.R. Evans and J.S. Coleman, Phys. Rev. Lett. 72, 1782 (1994).

11. T. Koike, T. Hara and S. Adachi, Phys Rev. Lett. 74, 5170 (1995).

12. D.J. Kaup, Phys. Rev. 172, 1331 (1968).

13. R. Ruffini and S. Bonnazzola, Phys. Rev. 187, 1767 (1969).

14. M. Colpi, S.L. Shapiro, and I. Wasserman, Phys. Rev. Lett. 57, 2485 (1986).

15. P. Jetzer, Phys. Rep. 220, 163 (1992).

16. E.W. Mielke and F.E. Schunck. gr-qc/9801063 (1998).

17. M. Gleiser and R. Watkins, Nucl. Phys. B 319, 733 (1989).

18. E. Seidel and W.-M. Suen, Phys. Rev. Lett. 72, (1994).

19. M. Colpi, S.L. Shapiro and S.A. Teukolsky, Astrophys. J. 369, 422 (1991). 